\title{
Großgefäßvaskulitiden: Mit gebündeltem Wissen gegen Folgeschäden
}

Sehstörungen, Erblindung, Schlaganfall - die Komplikationen einer Großgefäßvaskulitis können gravierend sein. Um Folgeschäden zu verhindern, muss die Gefäßentzündung früh erkannt und konsequent behandelt werden. Im Idealfall geschieht dies durch ein interdisziplinäres Ärzteteam. Doch welche diagnostischen Möglichkeiten gibt es und wie sollte die fachübergreifende Therapie aussehen? Den aktuellen Stand des Wissens hierzu haben Experten nun in einer deutschsprachigen Leitlinie zusammengefasst.

Die S2k-Leitlinie zum Management der Großgefäßvaskulitiden haben Vertreter aller beteiligten Fachrichtungen sowie Patientenvertreter unter Federführung der Deutschen Gesellschaft für Rheumatologie e. V. (DGRh) erarbeitet. Besorgniserregend sind v.a. die Komplikationen, die die Gefäßentzündung mit sich bringen kann. Dabei zählen die Sehstörungen, die bis zur Erblindung reichen können, zu den frühen Symptomen. Die neue Leitlinie empfiehlt daher, bereits beim Verdacht auf eine 
Riesenzellarteriitis umgehend mit einer Glukokortikoid-Therapie zu beginnen, die Augenschäden meist effektiv verhindert. Die Diagnostik sollte dann jedoch möglichst rasch vervollständigt werden, bevor die Gefäßveränderungen sich zu stark zurückbilden und nicht mehr sicher zu erkennen sind.

Die Diagnose der Riesenzellarteriitis stützt sich hauptsächlich auf bildgebende Verfahren, eine Biopsie sieht die Leitlinie nur noch in Ausnahmefällen vor. „Mithilfe von hochauflösendem Ultraschall, MRT- oder PETCT-Aufnahmen der Gefäße kann die Erkrankung heute meist sehr schnell diagnostiziert werden“, sagt Dr. Jan Henrik Schirmer von der Klinik für Innere Medizin I des UKSH, Campus Kiel, Erstautor der Leitlinie. Im Idealfall geschehe dies im Rahmen einer Fast-Track- beziehungsweise Akut-Sprechstunde in einem spezialisierten Zentrum hier könne eine Riesenzellarteriitis meist innerhalb von 24 Stunden sicher erkannt und die Therapie entsprechend früh eingeleitet werden.

Insgesamt umfasst die neue S2k-Leitlinie 22 spezielle Empfehlungen zu Diagnose, Behandlung und Verlaufskontrolle der Großgefäßvaskulitiden. „Die Leitlinie stimmt sehr gut mit den kürzlich publizierten europäischen Empfehlungen überein“, sagt Prof. Hendrik Schulze-Koops, Leiter der Rheumaeinheit am Klinikum der LMU München und Präsident der DGRh. Allerdings enthalte sie wesentlich detailliertere Empfehlungen für die klinische Praxis. So würden etwa genaue Richtwerte für die Dosierung und Dauer der Glukokortikoidgabe genannt, die sich nach der jeweiligen Krankheitsaktivität richteten. Damit solle Rückfällen entgegengewirkt werden, aber auch unerwünschten Folgen der Glukokortikoidtherapie wie Osteoporose, Diabetes, Grauem Star und - bei Patienten mit starkem Befall der Aorta einem gefährlichen Aortenaneurysma. „Die alleinige Therapie mit hochdosierten Glukokortikoiden hat zu Beginn der Behandlung noch immer ihren Platz", sagt SchulzeKoops. Das Therapiespektrum sei jedoch in den letzten Jahren um andere Wirkstoffe erweitert worden, die nun auch Eingang in die Leitlinie gefunden hätten. Viele Patienten könnten nun von einer Kombinationstherapie profitieren, die es erlaube, die Glukokortikoiddosis rascher zu senken, die Ge- fahr von Nebenwirkungen zu minimieren und dennoch Rückfälle zu vermeiden.

Nach einer Pressemitteilung der DGRh 